\title{
Semiclassical Coulomb Excitation Matrix Elements
}

\author{
B.V. Carlson, \\ Departamento de Física, Instituto Tecnológico de Aeronáutica - CTA, 12228-900, São José dos Campos, SP, Brazil \\ L.F. Canto, \\ Instituto de Física da Universidade do Rio de Janeiro, CP 68528, 21945-970, Rio de Janeiro, RJ, Brazil
}

and M.S. Hussein

Instituto de Física, Universidade de São Paulo, CP 66318, 05389-970, São Paulo, SP, Brazil

Received on 3 October, 2003

\begin{abstract}
Coulomb excitation matrix elements are often expressed in terms of the scalar electric potential and the electromagnetic fields. We show that, through an appropriate gauge transformation, the excitation matrix elements can always be expressed in terms of the electromagnetic fields alone. This change in representation becomes important when the widths of the excited states are taken into account.
\end{abstract}

\section{Introduction}

In an often cited work on Coulomb excitation[1] and in a more recent analysis of the same topic[2], the Coulomb excitation matrix elements are expressed in terms of the scalar electric potential and the electromagnetic fields. This representation of the interaction is adequate for use in a perturbative treatment and also provides a reasonably good estimate of the excitation cross section for states of zero width. In fact, in these two cases, the results are almost identical to those obtained by directly using the electromagnetic fields, or their derivatives, as these would appear in a classical treatment of the problem.

However, the mixed potential/field representation of the interaction is not adequate for a nonperturbative treatment of of excited states of finite width. In this case, the mixed representation yields large, unphysical cross sections due to the absorption of flux after excitation by the long-range potential term. The excitation cross section of a dipole transition, in particular, diverges in this case. This makes a treatment of multiple Coulomb excitation incorporating fluctuations contributions, such as those of the Brink-Axel type[3, 4, 5, 6, 7], impossible in the mixed representation, since finite widths are a fundamental component of such models.

In the following, we show that, through an appropriate gauge transformation, the Coulomb excitation matrix elements can always be expressed in terms of the electromagnetic fields alone. Aside from making a satisfying parallel with the classical case, the pure field representation of the interaction matrix elements is found to provide physically reasonable cross sections[7].

\section{Expanding the interaction Hamilto- nian}

The electromagnetic interaction Hamiltonian is given by

$$
V(t)=\int d^{3} x\left(\rho(\vec{x}, t) \varphi(\vec{x}, t)-\frac{1}{c} \vec{J}(\vec{x}, t) \cdot \vec{A}(\vec{x}, t)\right),
$$

where $\varphi(\vec{x}, t)$ and $\vec{A}(\vec{x}, t)$ are the scalar and vector electromagnetic potentials, for which

$$
\vec{E}(\vec{x}, t)=-\nabla \varphi(\vec{x}, t)-\frac{1}{c} \frac{\partial \vec{A}(\vec{x}, t)}{\partial t}
$$

and

$$
\vec{B}(\vec{x}, t)=\nabla \times \vec{A}(\vec{x}, t),
$$

and we assume $\rho(\vec{x}, t)$ and $\vec{J}(\vec{x}, t)$ to be the charge and current density of a nucleus. We assume that the source of the electromagnetic field does not overlap the nucleus.

We want to obtain the first few terms contributing to the energy in the expansion of the electromagnetic fields about the center of the nucleus, $\vec{x}=0$. Such an expansion is reasonable if the electromagnetic fields are slowly varying over the extent of the nucleus[8] We thus take

$$
\begin{aligned}
\int d^{3} x \rho(\vec{x}, t) \varphi(\vec{x}, t) \approx \int & d^{3} x \rho(\vec{x}, t)\left(\varphi_{0}(t)+\vec{x} \cdot \nabla \varphi_{0}(t)\right. \\
& \left.+\frac{1}{2} \vec{x} \vec{x} \cdot \nabla \nabla \varphi_{0}(t)+\cdots\right)
\end{aligned}
$$

and

$$
\begin{aligned}
\int d^{3} x \vec{J}(\vec{x}, t) \cdot \vec{A}(\vec{x}, t) \approx & \int d^{3} x \vec{J}(\vec{x}, t) \cdot \\
& \left(\vec{A}_{0}(t)+\vec{x} \cdot \nabla \vec{A}_{0}(t)+\cdots\right),
\end{aligned}
$$


where the subscript 0 on the fields and their derivatives denotes the evaluation of these at the point $\vec{x}=0$.

Evaluation of the scalar potential terms is straightforward. Evaluation of the vector potential terms requires a bit more work. We use the continuity equation,

$$
\nabla \cdot \vec{J}+\frac{\partial \rho}{\partial t}=0
$$

to derive two supplementary identities:

$$
\begin{aligned}
\int d^{3} x J_{k} & =\int d^{3} x\left(\nabla \cdot\left(x_{k} \vec{J}\right)-x_{k} \nabla \cdot \vec{J}\right) \\
& =\int d^{3} x x_{k} \frac{\partial \rho}{\partial t},
\end{aligned}
$$

where the integral of the exact divergence is zero due to the finite extent of $\vec{J}$, and

$$
\begin{aligned}
\int d^{3} x J_{k} x_{i} & =\int d^{3} x\left(\nabla \cdot\left(x_{k} \vec{J}\right) x_{i}-x_{k} x_{i} \nabla \cdot \vec{J}\right) \\
& =\int d^{3} x\left(-x_{k} \vec{J} \cdot \nabla x_{i}+x_{k} x_{i} \frac{\partial \rho}{\partial t}\right) \\
& =-\int d^{3} x J_{i} x_{k}+\int d^{3} x x_{k} x_{i} \frac{\partial \rho}{\partial t}
\end{aligned}
$$

which we rewrite as

$$
\int d^{3} x\left(J_{k} x_{i}+J_{i} x_{k}\right)=\int d^{3} x x_{k} x_{i} \frac{\partial \rho}{\partial t} .
$$

Using the first of these, we can write

$$
\begin{aligned}
\int d^{3} x \vec{J}(\vec{x}, t) \cdot \vec{A}_{0}(t) & =\sum_{k} \int d^{3} x J_{k}(\vec{x}, t) A_{0 k}(t) \\
& =\sum_{k} \int d^{3} x \frac{\partial \rho}{\partial t} x_{k} A_{0 k}(t) \\
& =\int d^{3} x \frac{\partial \rho}{\partial t} \vec{x} \cdot \vec{A}_{0}(t)
\end{aligned}
$$

Using the second, we find

$$
\begin{aligned}
& \int d^{3} x= \vec{J}(\vec{x}, t) \cdot(\vec{x} \cdot \nabla) \vec{A}_{0}(t)= \\
&= \sum_{i, k} \int d^{3} x J_{k}(\vec{x}, t) x_{i} \partial_{i} A_{0 k}(t) \\
&= \frac{1}{2} \sum_{i, k} \int d^{3} x\left(\left(J_{k} x_{i}+J_{i} x_{k}\right) \partial_{i} A_{0 k}\right. \\
&\left.\quad+\left(J_{k} x_{i}-J_{i} x_{k}\right) \partial_{i} A_{0 k}\right) \\
&=\frac{1}{2} \int d^{3} x \frac{\partial \rho}{\partial t} \vec{x} \cdot(\vec{x} \cdot \nabla) \vec{A}_{0}(t) \\
& \quad+\frac{1}{2} \int d^{3} x \vec{J} \cdot\left((\vec{x} \cdot \nabla) \vec{A}_{0}-\nabla\left(\vec{x} \cdot \vec{A}_{0}\right)\right),
\end{aligned}
$$

where the operator $\nabla$ acts only the vector potential $\overrightarrow{A_{0}}$ in the last term. We can manipulate the integrand of the second term further,

$$
\begin{aligned}
\vec{J} \cdot\left((\vec{x} \cdot \nabla) \vec{A}_{0}-\nabla\left(\vec{x} \cdot \vec{A}_{0}\right)\right) & =-\vec{J} \cdot\left[\vec{x} \times\left(\nabla \times \vec{A}_{0}\right)\right] \\
& =(\vec{x} \times \vec{J}) \cdot\left(\nabla \times \overrightarrow{A_{0}}\right),
\end{aligned}
$$

so that we may finally write for the entire term,

$$
\begin{aligned}
\int d^{3} x \quad & \vec{J}(\vec{x}, t) \cdot(\vec{x} \cdot \nabla) \vec{A}_{0}(t)= \\
= & \frac{1}{2} \int d^{3} x \frac{\partial \rho}{\partial t} \vec{x} \cdot(\vec{x} \cdot \nabla) \vec{A}_{0}(t) \\
& +\frac{1}{2} \int d^{3} x(\vec{x} \times \vec{J}) \cdot\left(\nabla \times \vec{A}_{0}(t)\right) .
\end{aligned}
$$

Putting all the pieces together, we have

$$
\begin{aligned}
V(t)= & \int d^{3} x\left(\rho(\vec{x}, t) \varphi(\vec{x}, t)-\frac{1}{c} \vec{J}(\vec{x}, t) \cdot \vec{A}(\vec{x}, t)\right) \\
\approx & \int d^{3} x \rho(\vec{x}, t)\left(\varphi_{0}(t)+\vec{x} \cdot \nabla \varphi_{0}(t)\right. \\
& \left.+\frac{1}{2} \vec{x} \vec{x} \cdot \nabla \nabla \varphi_{0}(t)+\cdots\right) \\
& -\frac{1}{c} \int d^{3} x \frac{\partial \rho}{\partial t} \vec{x} \cdot\left(\vec{A}_{0}(t)+\frac{1}{2}(\vec{x} \cdot \nabla) \vec{A}_{0}(t)+\cdots\right) \\
& -\frac{1}{2 c} \int d^{3} x(\vec{x} \times \vec{J}) \cdot\left(\nabla \times \vec{A}_{0}(t)\right)+\cdots
\end{aligned}
$$

We can write the second line in this expansion as

$$
\begin{array}{r}
-\frac{1}{c} \int d^{3} x \frac{\partial \rho}{\partial t} \vec{x} \cdot\left(\vec{A}_{0}(t)+\frac{1}{2}(\vec{x} \cdot \nabla) \vec{A}_{0}(t)+\cdots\right) \\
=-\frac{1}{c} \int d^{3} x \frac{\partial \rho}{\partial t} \int_{0}^{\vec{x}} \vec{A}(\vec{l}, t) \cdot d \vec{l} .
\end{array}
$$

\section{The Gauge Transformation}

Let us define a gauge transformation $\Lambda(\vec{x}, t)$ as

$$
\Lambda(\vec{x}, t)=-\int_{0}^{\vec{x}} \vec{A}(\vec{l}, t) \cdot d \vec{l}
$$

This gauge transformation will annul the integral above, since for $\overrightarrow{A^{\prime}}(\vec{x}, t)=\vec{A}(\vec{x}, t)+\nabla \Lambda(\vec{x}, t)$,

$$
\int_{0}^{\vec{x}} \overrightarrow{A^{\prime}}(\vec{l}, t) \cdot d \vec{l}=\int_{0}^{\vec{x}} \vec{A}(\vec{l}, t) \cdot d \vec{l}+\Lambda(\vec{x}, t)=0 .
$$

It will not modify the magnetic field, since $\nabla \times \nabla \Lambda(\vec{x}, t)=$ 0 . It will modify the scalar potential, however, which now becomes

$$
\varphi^{\prime}(\vec{x}, t)=\varphi(\vec{x}, t)-\frac{1}{c} \frac{\partial \Lambda}{\partial t}=\varphi(\vec{x}, t)+\frac{1}{c} \frac{\partial}{\partial t} \int_{0}^{\vec{x}} \vec{A}(\vec{l}, t) \cdot d \vec{l} .
$$

Expanding the transformed scalar potential about $\vec{x}=0$, we find

$$
\begin{aligned}
\varphi^{\prime}(\vec{x}, t)= & \varphi(\vec{x}, t)+\frac{1}{c} \frac{\partial}{\partial t} \int_{0}^{\vec{x}} \vec{A}(\vec{l}, t) \cdot d \vec{l} \\
\approx & \varphi_{0}(t)+\vec{x} \cdot\left(\nabla \varphi_{0}(t)+\frac{1}{c} \frac{\partial \vec{A}_{0}}{\partial t}\right) \\
& +\frac{1}{2} \vec{x} \vec{x} \cdot \nabla\left(\nabla \varphi_{0}(t)+\frac{1}{c} \frac{\partial \vec{A}_{0}}{\partial t}\right)+\cdots
\end{aligned}
$$


so that, after the gauge transformation, we have for the interaction,

$$
\begin{array}{r}
V(t) \approx \int d^{3} x \rho(\vec{x}, t)\left(\varphi_{0}(t)+\vec{x} \cdot\left(\nabla \varphi_{0}(t)+\frac{1}{c} \frac{\partial \vec{A}_{0}}{\partial t}\right)\right. \\
\left.+\frac{1}{2} \vec{x} \vec{x} \cdot \nabla\left(\nabla \varphi_{0}(t)+\frac{1}{c} \frac{\partial \vec{A}_{0}}{\partial t}\right)+\cdots\right) \\
-\frac{1}{2 c} \int d^{3} x(\vec{x} \times \vec{J}) \cdot\left(\nabla \times \vec{A}_{0}(t)\right)+\cdots
\end{array}
$$

and

the center of the charge/current distribution of interest. We then have

$$
\begin{gathered}
\varphi(\vec{b}, z, t)=\gamma \frac{Z e}{\sqrt{\left(\vec{b}-\vec{b}_{0}\right)^{2}+\gamma^{2}(z-v t)^{2}}} \\
\vec{A}(\vec{b}, z, t)=\frac{v}{c} \hat{z} \varphi(\vec{b}, z, t) .
\end{gathered}
$$

The electric field that results is

$$
\vec{E}_{0 \perp}(t)=-\gamma \vec{b}_{0} \frac{Z e}{\left(\vec{b}_{0}^{2}+(\gamma v t)^{2}\right)^{3 / 2}}
$$

and

$$
E_{0 \| \mid}(t)=-\gamma v t \frac{Z e}{\left(\vec{b}_{0}^{2}+(\gamma v t)^{2}\right)^{3 / 2}},
$$

which, together with the matrix elements of the dipole operator $\vec{p}$, are all we need to describe excitations of the giant dipole resonance. Matrix elements for other multipolarities can be calculated similarly.

Bayman and Zardi[9] have noted that the mixed potential representation of the interaction matrix elements of Refs. [1] and [2] also neglects relativistic contributions to the quadrupole and higher multipolarity matrix elements that could become important at high energies. These are also taken into account correctly (and automatically) when the pure field representation is used.

$\mathrm{BVC}$ and MSH acknowledge support from FAPESP. The authors acknowledge partial support from the CNPq.

\section{References}

$$
q=\int d^{3} x \rho(\vec{x}, t)
$$

$\vec{p}$ is the eletric dipole operator,

$$
\vec{p}=\int d^{3} x \vec{x} \rho(\vec{x}, t),
$$

the $Q_{i j}$ are the traceless electric quadrupole operators,

$$
Q_{i j}=\int d^{3} x\left(x_{i} x_{j}-\vec{x}^{2} \delta_{i j} / 3\right) \rho(\vec{x}, t),
$$

and $\vec{m}$ is the magnetic dipole operator,

$$
\vec{m}=\frac{1}{2 c} \int d^{3} x \vec{x} \times \vec{J}(\vec{x}, t) .
$$

\section{Conclusions}

As an example, we take as the potentials those due to a relativistic nucleus of charge $Z$ passing on a straight-line trajectory with velocity $v$ in the $\hat{z}$ direction at a distance $b_{0}$ from
[1] C.A. Bertulani, L.F. Canto, M.S. Hussein, and A.F.R. de Toledo Piza, Phys. Rev. C53, 334 (1996).

[2] H. Esbensen and C.A. Bertulani, Phys. Rev. C65, 24605 (2002).

[3] B.V. Carlson, M.S. Hussein, A.F.R. de Toledo Piza, and L.F. Canto, Phys. Rev. C60, 14604 (1999).

[4] B.V. Carlson and M.S. Hussein, Phys. Rev. C59, R2343 (1999).

[5] B.V. Carlson, L.F. Canto, S. Cruz-Barrios, M.S. Hussein, and A.F.R. de Toledo Piza, Ann. Phys. (New York) 276111 (1999).

[6] L.F. Canto, B.V. Carlson, M.S. Hussein, and A.F.R. de Toledo Piza, Phys. Rev. C60, 64624 (1999).

[7] M.S. Hussein, B.V. Carlson, L.F. Canto, and A.F.R. de Toledo Piza, Phys. Rev. C66 34615 (2002).

[8] J.D. Jackson, Classical Electrodynamics, J. Wiley and Sons, Inc., New York, 1962).

[9] B.F. Bayman and Z. Zardi, Los Alamos Preprint Archives, nucl-th/0210060 (2001). 